\title{
CUÑAS MOTRICES BILINGÜES
}

\author{
Bilingual motor wedges \\ Cunhas motrizes bilíngües \\ Luisa Núñez Alonso (1) \\ Ángel Pérez Pueyo (2) \\ Izaskun Urrutia Velásquez (3) \\ Elena Núñez Alonso (4) \\ David Hortigüela Alcalá (5) \\ Alejandra Hernando Garijo (6) \\ Jorge Garrote García (7) \\ Patricia Rivera Blanco (8)
}

(1) Academia “Actitud Bilingüe”, +34 987076607 actitudbilingue@gmail.com

(2) Universidad de León, +34 619867767 angel.perez.pueyo@unileon.es

(3) Academia “Actitud Bilingüe”, +34 987076607 izaurrutia@gmail.com

(4) CEIP “Quevedo”, León, +34987076607 enua0000@yahoo.com

(5) Facultad de Educación. Universidad de Burgos, +34 947259517

dhortiguela@ubu.es

(6) Facultad de Educación. Universidad de Burgos, +34 649690858 ahgarijo@ubu.es.

(7) IES Lancia (Leon) +34 665016492 jorge.garrote@hotmail.com

(8) IES Gaspar Melchor de Jovellanos, Fuenlabrada. Teléfono: +34 916971565.

patriciarivbla@gmail.com

\begin{abstract}
Resumen
La experiencia presenta el proceso de diseño de una propuesta de "cuñas motrices bilingües” generadas en un contexto extraescolar por miembros del grupo Actitudes para su posterior aplicación en el contexto escolar. Éstas relacionan las cuñas motrices que se llevan a la práctica en el contexto escolar y la secuenciación de competencias de la competencia lingüística en lengua extranjera del proyecto INCOBA. Se han utilizado ciclos de investigación-acción para generar experiencias que puedan transferirse al Las cuñas motrices bilingües


contexto escolar, por lo que el grupo de trabajo cuenta con profesorado en activo para el diseño de las mismas. Los resultados demuestran gran nivel de participación del alumnado y un nivel de satisfacción alto de los mismos y de sus familias.

Palabras clave: Cuñas motrices bilingües; proyecto INCOBA; secuenciación de competencias; lengua extranjera

\begin{abstract}
The experience presents the process of designing a proposal for "bilingual driving wedges" generated in an out-of-school context by members of the Actitudes group for later application in the school context. These relate the driving wedges that are put into practice in the school context and the sequencing of competences of the foreign language proficiency of the INCOBA project. For its design, research-action cycles have been used with the intention of generating experiences that can be transferred to the school context, so that the working group has active teaching staff for their design. The results demonstrate the high level of student participation and a high level of satisfaction of the students and their families.
\end{abstract}

Keywords: Bilingual motor wedges; INCOBA project; sequencing of skills/competencies; foreign language

\title{
Resumo
}

A experiência mostra o processo de concepção de uma proposta de "cunhas motrizes bilíngües" gerados em um contexto formal, por membros das atitudes do grupo para posterior aplicação no contexto escolar. Estes se relacionam com as cunhas motrizes bilíngües que estão sendo implementadas no contexto escolar e sequenciamento habilidades de proficiência em língua estrangeira do projeto INCOBA. Têm sido usados pesquisa-ação com a intenção de criar experiências que podem ser transferidos para o contexto escolar, de modo que o grupo de trabalho tem professores ativos para projetarlos. Os resultados demonstram o alto nível de participação dos alunos e um alto nível de satisfação de si e suas famílias.

Palavras-chave: Cunhas motrizes bilíngües; projeto INCOBA; habilidades de seqüenciamento; língua estrangeira 


\section{Introducción}

La incorporación de la educación bilingüe en España es una realidad, aunque el cuestionamiento y la crítica a la misma es constante (Fernández-Barrionuevo, 2009). Sin embargo, son numerosos los autores que destacan sus beneficios para el desarrollo del aprendizaje así como para el desarrollo integral de los alumnos.

El grupo Actitudes plantea una propuesta de secuenciación de competencias a través del “Proyecto INCOBA” (Pérez-Pueyo, et al., 2013), en este caso en relación a la competencia lingüística en lengua extranjera (LE) (Hernando, Hortigüela, Garrote \& Blanco, 2016), a través de la cual se propone contribuir a esta competencia desde diferentes materias de manera coordinada.

En contextos no bilingües, Vaca (2007) y Cebrián, Martín y Arroyo (2013) plantean la utilización de las “cuñas motrices”. Éstas son actividades motrices realizadas entre otras dos que requieran un mayor nivel de concentración, utilizando los materiales y dentro del aula, consiguiendo “dar salida a la necesidad de movimiento de los niños y trabajar contenidos motrices o bien contenidos de otros ámbitos a través del movimiento (lecto-escritura, idiomas, pensamiento lógico-matemático, conocimiento del medio, música, ritmo, etc.)” (Cebrián, Martín, \& Arroyo, 2013, p.13). Además, Amor y Pascual (2012) destacan que la actividad física puede influir decisivamente al desarrollo de las destrezas lingüísticas del alumnado. Por todo ello, esta experiencia presenta una hibridación de ambas propuestas a través de las cuñas motrices bilingües (CMB) diseñadas en el laboratorio lingüístico del Grupo Internivelar e Interdisciplinar Actitudes.

\section{Contextualización}

El laboratorio lingüístico se lleva a cabo en la academia “Actitud Bilingüe” de León. El equipo docente está formado por varios filólogos ingleses, profesores nativos, profesores de primaria y especialistas en inglés, que diseñan y ponen en práctica CMB en actividades extraescolares con alumnos de primaria agrupados en horquillas de dos en dos años.

\section{Diseño y desarrollo}

A través de ciclos de investigación-acción sencillos (Fraile, 1995), se plantean las siguientes fases: 
Fase 1.- La hibridación conceptual. Ésta se realiza a través de la integración de: a) la secuenciación de la competencia en LE del Grupo Actitudes, basada en la concreción del currículo de inglés en relación a las cuatro habilidades para el desarrollo de la LE (Comprensión oral, Expresión oral, Compresión escrita y Expresión escrita) (figura 1) pudiendo ver un ejemplo de indicador de la dimensión Expresión oral en la Tabla 1; y b) las cuñas motrices.

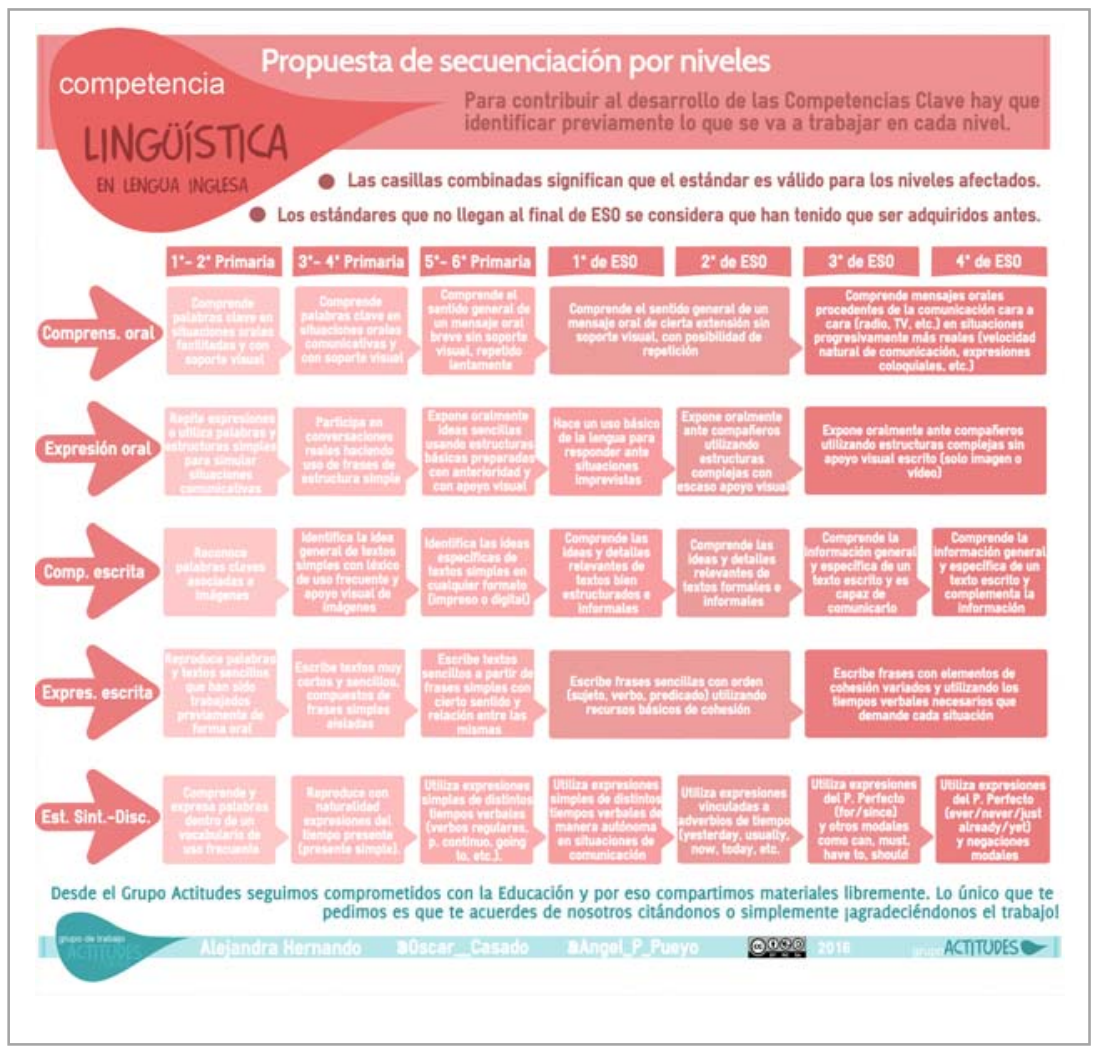

Figura 1.

Infografía resumen de la secuenciación del Grupo Actitudes de la competencia lingüística en LE

Fase 2.- Diseño de CMB. Éstas tendrán una duración variable de 5 a 10 minutos al igual que las cuñas motrices (Cebrián, Martín, \& Arroyo, 2013; Vaca, 2007). Ejemplo de la misma puede ser el siguiente CMB: Los alumnos, de dos en dos y al azar, sacan sus nombres de una caja. Luego deben simular que se encuentran por la calle (dramatizar), saludarse y preguntarse cómo se encuentran de manera amable. También debe preguntarse por una situación de las cotidianas, trabajadas en clase de inglés y que les tocará al azar (tirando un dado que en cada cara tiene una situación). Además, los alumnos pueden optar por utilizar frases hechas que podrán utilizar de apoyo o preguntar por otra persona identificándola con descripciones básicas o datos personales. Como se puede comprobar en la tabla 1 , los aspectos que se describen en la Las cuñas motrices bilingües 
CMB se refieren a la subcompetencia o indicador establecido para el $3^{\circ}$ y $4^{\circ}$ cursos de primaria

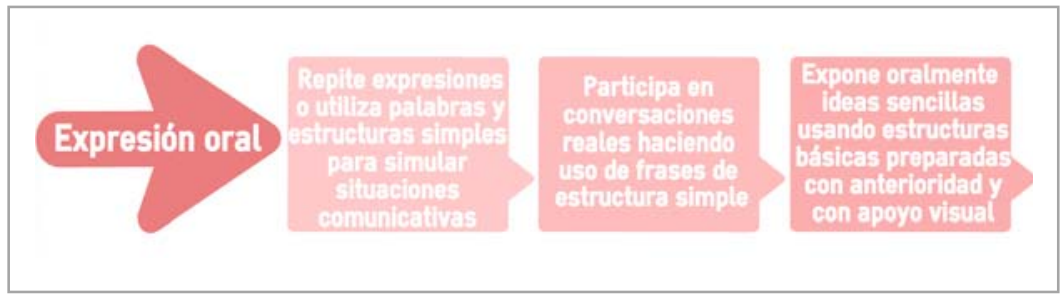

Figura 1.

Ejemplo de indicador de la dimensión de expresión oral de la competencia lingüística en LE (Hernando, Hortigüela, Garrote, \& Blanco, 2016)

Fase 3.- Puesta en práctica. Se ha llevado a cabo durante el curso 2016-2017 en sesiones de una hora por parte de dos profesoras (una filóloga inglesa con un nivel C2 y una nativa de origen inglés). Estas imparten media hora cada una, pues los grupos se encuentran duplicados y las dos profesoras se intercambian a media clase.

Aunque en ambos casos las profesoras utilizan actividades de carácter manipulativo, es una de ellas, cada día, la que en la sesión experimenta con actividades que requieren levantarse de la mesa para utilizar un espacio diferente pero dentro del aula; como ocurrirá en las de los centros escolares. Es en esta parte en la que participa la observadora externa tomando notas para su análisis posterior.

Fase 4.- El análisis de los resultados. La observadora externa, con una lista de control en la que realiza observaciones (López-Pastor, 2006), toma notas de los comportamientos y resultados producidos tanto de los alumnos como de la profesora. En ambas aulas existe una ventana hacia el pasillo que permite realizar una observación externa. Toda la información obtenida, tanto por las profesoras como por la observadora externa permite realizar procesos posteriores de revisión, corrección y nueva propuesta de aquellas CMB que lo necesiten.

Fase 5.- Trasferencia a centro escolares. Esta fase se llevará a cabo el próximo curso por docentes en activo del Grupo Actitudes que desarrollan su labor en centros de primaria

\section{Evaluación y conclusiones}

La puesta en práctica durante los dos primeros trimestres del curso, tanto de los niños como de sus familias que han sido entrevistadas, ha permitido comprobar que el grado de satisfacción de ambos es alto. Las familias reconocen que sus hijos asisten a clase 
muy contentos y demuestran en casa los aprendizajes adquiridos. Los niños valoran positivamente las sesiones y las cuñas en particular a través de un sistema de semáforo que planteamos al salir de cada sesión. En la actualidad continuamos elaborando una serie de CMB para poder ofrecer a los docentes de los diferentes cursos.

En los centros bilingües se suele utilizar la metodología CLIL/AICLE, al centrarse en integrar y combinar el conocimiento social, cultural y artístico que se desprende de las materias bilingües (Hernando, Hortigüela \& Pérez-Pueyo, 2016). Las CMB pretenden poder integrarse tanto en las áreas bilingües como no bilingües para complementar el proceso de aprendizaje de la LE.

\section{Referencias}

Amor, T., \& Pascual, L. F. (2012). Actividades bilingües en Educación Física de base. Educación Física y bilingüísmo. Madrid: CCS.

Fernández-Barrionuevo, E. (2009). Crítica sobre el trabajo en grupos en EF bilingüe: consideraciones teóricas y aplicaciones prácticas. Innovación y experiencias educativas, 20, 1-19.

Fraile, A. (1995). La investigación-acción: instrumento de formación para el profesorado de educación física. Apunts: Educación Física y Deportes, (42), 4652.

Hernando, A., Hortigüela, D., \& Pérez-Pueyo, A. (2018). Percepciones de los coordinadores de los programas bilingües y docentes de educación física en inglés en secundaria sobre el proceso de implantación y desarrollo del bilingüismo en la Comunidad de Castilla y León. Retos, (33), 63-68.

Hernando, A., Hortigüela, D., Garrote, J., \& Blanco, P. (2016). Secuenciación de la competencia lingüística en lengua extranjera. Recuperado de http://www.grupoactitudes.com/competencia-lingsitica-ingls

Hortigüela, D., Hernando, A., \& Pérez-Pueyo, A. (2016). ¿Qué estrategias metodológicas utiliza el profesor de Educación Física bilingüe en el aula? En M.I. Amor, J.L Luengo-Almena \& M. Martínez (coords.), Educación intercultural: metodología de aprendizaje en contextos bilingües (pp. 103-106). Granada: Atrio. 
López-Pastor, V. M. (Coord.) (2006). La Evaluación en Educación Física: Revisión de los modelos tradicionales y planteamiento de una alternativa: La evaluación formativa y compartida. Buenos Aires: Miño y Dávila.

Cebrián, B., Martín, M. I., \& Arroyo, M. A. (2013). Cómo trabajar la motricidad en el aula. Cuñas motrices para infantil y primaria. Madrid: Miño y Dávila.

Pérez-Pueyo, A., Heras, C., Barba, J.J., Casado, O., Vega, D., \& Pablos, L. (2013). Qué son las Competencias Básicas y cómo se trabajan en España. Diferentes maneras de llevarlas de la teoría al aula. Hacia el Proyecto INCOBA. León: Actitudes Profesionales.

Vaca, M. (2007). Un proyecto para una escuela con cuerpo y en movimiento. Ágora para la EF y el Deporte, 4(5), 91-110. 